\title{
VIOLENCIA DE GÉNERO Y MIGRACIÓN: MUJERES SOLICITANTES DE ASILO Y BAJO PROTECCIÓN INTERNACIONAL EN MÉXICO
}

GENDER VIOLENCE AND MIGRATION: WOMEN ASYLUM-SEEKERS AND UNDER INTERNATIONAL PROTECTION IN MEXICO

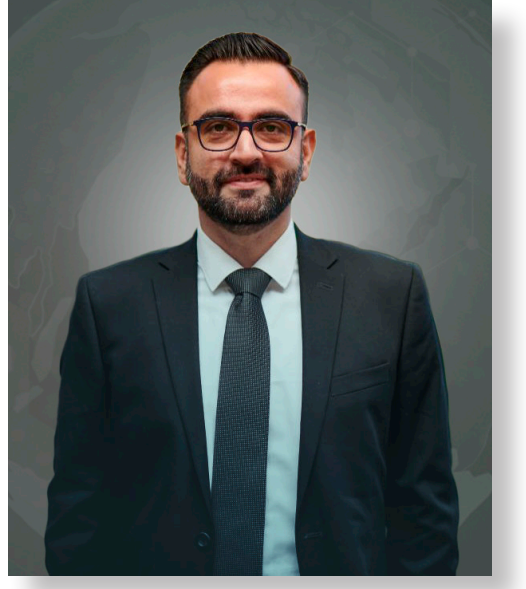

Francisco J. Landeros Jaime

Doctor en Sociología por la Pontificia Universidad Católica de Chile

Clínica Jurídica-Instituto para las Mujeres en la

Migración (IMUMI), México

clinicajuridica@imumi.org

ORCID: https://orcid.org/0000-0001-5497-3666

México

DOI: https://doi.org/10.5377/umhs.v2i1.12998

Recibido: 21 de julio de 2021

Aceptado: 12 de noviembre de 2021

\section{RESUMEN}

Los flujos migratorios de los últimos años en México han registrado un aumento significativo, y con ello la violencia se ha convertido en una constante para las personas migrantes. El objetivo de este artículo es identificar los tipos de violencia que sufren las mujeres solicitantes de asilo y que son sujetas a alguna figura de protección internacional en territorio mexicano; asimismo, se analizan las relaciones que se presentan entre las distintas violencias. Desde una perspectiva metodológica mixta, se integra la precisión del dato numérico con la sensibilidad de lo cualitativo, y se retoman los testimonios de mujeres originarias principalmente de países del triángulo norte de Centroamérica. Los principales hallazgos indican que las mujeres en la migración no resuelven sus problemas vinculados a la persecución y violencia con el hecho de salir de su lugar de origen y cruzar una frontera internacional, sino que las mismas prácticas violentas, principalmente físicas y psicológicas de las que fueron víctimas, les persiguen hasta el lugar de destino.

PALABRAS CLAVE: Migración; Violencia de género; Mujeres migrantes.

\section{ABSTRACT}

The migratory flows during the recent years in Mexico have registered a significant increase, alongside it the violence that has become in a constant situation for migrant people. This article aims to identify what kind of violence suffer the asylum seeker women and who are subject to some international protection figures in Mexican territory. Also, this document presents an analysis of the relations among different kinds of violence. Using a mixed-method perspective that integrates the precision of numeric data with the sensitivity of qualitative information, the testimonies of Centroamerican women are taken to deepen on the experiences and interpretations presented by them. The main findings highlight that all the problems related to the violence and persecution that women face constantly are not solved leaving their homeland, instead of that, the same violent outbreaks, mainly physical and psychological, follow them to the country of destination.

KEYWORDS: Migration; Gender violence; Migrant women. 


\section{INTRODUCCIÓN}

La migración internacional en América Latina y el Caribe sigue registrando un incremento constante en sus flujos producto de la desigualdad propiciada por complicaciones políticas, sociales y económicas, mismas que ilustran sus impactos a partir de las brechas salariales y de acceso a servicios públicos para migrantes entre el país de origen y destino. Para el caso de México, dichas problemáticas se recrudecen con las prácticas violentas primordialmente ejercidas por parte de autoridades, pandillas y crimen organizado, en un contexto donde mujeres provenientes del triángulo norte de Centroamérica, son las principales víctimas. Cabe destacar que, en dichas dinámicas, la violencia se manifiesta de distintas formas, siendo la de carácter físico la más visible y de mayor recurrencia.

Según los datos demográficos de los últimos años, la llegada de personas que huyen de la violencia generada en los países de origen ha incrementado de manera sustancial, lo que se refleja en las solicitudes de personas buscando la protección que no han recibido en sus países de origen. Al respecto la Comisión Mexicana de Apoyo a Refugiados (COMAR) registró en 2019 al menos 70,418 solicitudes de asilo de personas originarias principalmente de Honduras, El Salvador y Cuba (Comisión Mexicana de Ayuda a Refugiados, 2021). Durante 2020 las solicitudes disminuyeron hasta 41,179 producto de la pandemia por Covid-19. No obstante, hasta mayo de 2021 se hablaba de 41,195 solicitudes de asilo en el país, lo que refleja un aumento en el flujo migratorio originado principalmente por la violencia generalizada en el lugar de origen (Torre Cantalapiedra, 2020; Torre Cantalapiedra et al., 2021; Torre Cantalapiedra \& Mariscal Nava, 2020). Es crucial puntualizar que las solicitudes de asilo en México son admisibles bajo la definición internacional de persona refugiada tomando en cuenta un motivo de persecución adicional a la Convención de 1951, y también bajo la acepción del concepto para Latinoamérica proveniente de la Declaración de Cartagena de 1984, es decir que México reconoce:

A quienes por fundados temores de ser perseguidos en sus países de origen buscan protección por alguno de los 6 motivos comprendidos que son: raza, religión, nacionalidad, pertenencia a determinado grupo social, opinión política y género (este motivo sólo en la ley mexicana).

A quienes han visto amenazada su vida, seguridad o libertad a causa de violencia generalizada, agresión extranjera, conflictos internos, violación masiva de los derechos humanos u otras circunstancias que hayan perturbado gravemente el orden público.

Porotrolado, esimportante señalarque México contempla varias figuras de protección internacional: 1) Condición de persona refugiada, 2) Asilo político, 3) Documento provisional por razones humanitarias; 4) Protección complementaria, 5) Declaratoria de Apátrida. De acuerdo con la necesidad de protección de la persona, la COMAR (Comisión Mexicana de Ayuda a Refugiados) o el INM (Instituto Nacional de Migración) o SRE (Secretaría de Relaciones Exteriores) reconocen la condición u otorgan alguna otra figura de protección a la solicitante. A partir de lo anterior, el objetivo principal de este artículo no solamente es identificar los distintos tipos de violencia que sufren las mujeres solicitantes de asilo y sujetas a protección internacional en territorio mexicano, sino también ahondar en las historias, experiencias e interpretaciones derivadas de la interacción entre actores involucrados.

En este artículo se incluyen los casos de 65 mujeres solicitantes de asilo que fueron atendidas 
desde agosto del 2020 hasta abril del 2021 por el equipo de la Clínica Jurídica del Instituto para las Mujeres en la Migración A.C. (IMUMI), siendo las principales fuentes para la obtención de datos e información, las conversaciones formales e informales, documentación oficial, y bitácoras de casos.

A partir del análisis de las variables y categorías temáticas, este artículo ilustra cómo la diversidad de dinámicas y prácticas violentas interactúan para crear nuevas representaciones que generan una transnacionalización de la violencia, lo que significa que, para las mujeres que participan en este trabajo, el miedo, maltrato de distintos tipos, preocupación y ansiedad no finaliza al dejar el lugar de origen, sino que les persigue en su vida diaria incluso en el sitio de acogida.

Esta modalidad extendida de la violencia añade mayor complejidad al desarrollo de los proyectos migratorios de las mujeres migrantes. Adicionalmente, algunos problemas relacionados a la xenofobia, racismo, discriminación basada en elementos étnicos, tales como lenguaje, creencias religiosas y aspecto físico; fueron identificados como parte del análisis, lo que representa también una ventana de oportunidad para investigaciones futuras dentro de la académica u organizaciones de la sociedad civil.

\section{METODOLOGÍA Y DATOS}

Para la obtención y análisis de datos de este artículo se diseñó una estrategia metodológica de corte mixto (Kuckartz, 2014; Rädiker \& Kuckartz, 2019) que consistió en documentar la información registrada por el equipo de la Clínica Jurídica del IMUMI a partir de la atención brindada a mujeres migrantes víctimas de violencia ya sea en el lugar de origen como en el destino, lo que sirvió para cuantificar e identificar frecuencias en torno a los distintos tipos de violencias. Asimismo, desde lo cualitativo se analizaron las principales problemáticas que las usuarias mencionaron haber enfrentado, no solamente durante su trayecto migratorio, sino también a lo largo de su vida.

\section{La primera etapa consistió en la} documentación de los casos que se atendieron durante el periodo de enero de 2020 a abril de 2020, donde las hojas de registro y bitácoras de atención fueron las principales fuentes de datos, también se incluyeron documentos formales e informales, mensajes a través de celular y conversaciones informales.

Con relación a los perfiles que se incluyen en la muestra, destaca que son 65 mujeres migrantes con necesidad de protección internacional cuya particularidad es que sean 1) sobrevivientes de violencia sexual en su país de origen o en tránsito por México; 2) Víctimas de violencia física, sexual, psicológica, doméstica, laboral o económica en su país de origen o en tránsito por México; 3) Víctimas de violencia institucional en México; 4) Víctimas de trata de personas en su país de origen o en tránsito por México; 5) Víctimas de planificación familiar forzada, mutilación genital forzada y discriminación por orientación sexual en su país de origen; 6) Con riesgo potencial de castigo en su país de origen por transgredir los valores o costumbres morales de su comunidad; 7) Con riesgo potencial en su país de origen de ser afectada por una Ley persecutoria.

A partir de la documentación generada se diseñó y construyó una matriz para análisis mixto con el software MAXQDA2020. Se diseñaron también los códigos vinculados a las categorías analíticas de interés y a la conceptualización de violencia construida para guiar el análisis.

La codificación permitió identificar elementos 
para ahondar en aspectos que son subyacentes en las prácticas violentas a las que son sometidas las mujeres migrantes. Por otro lado, es importante aclarar que las citas textuales que se incluyen en este artículo se retoman de forma íntegra desde el instrumento de registro que se utilizó para recabar los datos, se presenta de esta forma para lograr reflejar —en la mayor medida - la esencia de lo expresado por la usuaria.

Asimismo, es importante mencionar que para cada caso (65) se diseñaron también distintos tipos de variables que permitieran identificar de mejor manera las características socioculturales de las usuarias del IMUMI, mismas que son presentadas en este artículo a partir de las frecuencias registradas.

De esta forma, en la integración entre la precisión del dato cuantitativo con la profundidad de los testimonios y narrativas migrantes radica la relevancia metodológica de este estudio, pues no solamente analiza una parte del fenómeno, sino que lo aborda como una problemática social relacional donde cada método (cuantitativo y cualitativo) aporta elementos esenciales para entender los flujos migratorios actuales, lo que deriva en posibles propuestas de políticas públicas orientadas a mejorar la calidad de vida de las poblaciones migrantes en México.

\section{ANÁLISIS DE LOS RESULTADOS}

Los problemas que enfrentan las mujeres migrantes originarias principalmente de países del triángulo norte de Centroamérica generan diversas complicaciones a nivel social y económico, en un contexto en el que la violencia que les obligó a dejar su país de origen se recrudece a tal nivel que les es imposible consolidar su proyecto de vida migrante.

Si bien en la muestra de este artículo la violencia se presenta de formas diversas, son la de tipo físico y psicológico las de mayor frecuencia perpetradas ya sea en el lugar de origen o en el destino. A partir de lo anterior, es relevante subrayar la relación que se presenta entre los tipos de violencia teniendo como base los más recurrentes.

En ese sentido, en esta sección se presentan no solamente los hallazgos más relevantes en cuanto a la violencia física y psicológica ejercida en contra de mujeres migrantes originarias principalmente de países centroamericanos, sino también cómo se presentan las relaciones entre las distintas violencias, lo que a su vez genera escenarios de alta complejidad para las personas en movilidad y las organizaciones civiles que atienden particularmente este tipo de poblaciones en territorio mexicano.

De esta forma, es importante subrayar que las historias presentadas a partir de los registros generados por el equipo de la Clínica Jurídica del IMUMI se insertan en un ambiente violento y de vulnerabilidad producido por la pobreza que genera el carecer de un empleo estable y bien remunerado, redes de apoyo limitadas y problemáticas vinculadas a la discriminación, xenofobia, machismo y racismo, lo que se denomina también como "pobreza invisible" (Landeros Jaime, 2020).

\section{"Fui testigo del asesinato de mis hermanos; fue la M18"}

Entender la carga emocional con la que las mujeres migrantes llegan al lugar de destino es fundamental para ahondar en el análisis de las consecuencias derivadas de las experiencias violentas registradas a lo largo de su vida, donde las principales personas que las agreden forman parte de su propia estructura familiar, aunque también hay casos donde los victimarios son miembros de pandillas, grupos delincuenciales, instituciones 
policiacas e incluso migratorias. De esta forma, en la violencia ejercida en contra de las mujeres intervienen distintos actores en diversos contextos, lo que resulta de mayor complejidad al momento de entender cómo funcionan las relaciones de poder en torno al ejercicio de cada violencia en particular y cómo esta misma les persigue a las personas más allá de las fronteras.

Vine a México con mi familia huyendo del peligro en Honduras, porque fuimos atacados por personas con conexiones con el crimen organizado y las pandillas porque intentamos manejar un negocio y pedir ayuda al gobierno. Después de que intentamos buscar ayuda en la justicia, las amenazas de muerte en nuestra contra aumentaron y nuestros perseguidores incluso nos siguieron a México. La policía y los tribunales de nuestro país no nos ayudaban (E64, Comunicación personal, enero 2021).

El caso de E64 ${ }^{1}$ ilustra la forma en la que a partir de la violencia física se presentan otro tipo de complicaciones que a su vez registran una interconexión entre distintos actores clave. Es decir, la violencia física en los casos analizados marca la pauta para la generación de situaciones más complejas y violentas donde la intervención de autoridades de diferentes niveles ocasiona prácticas en torno al ejercicio de poder que potencializan los efectos negativos en los trayectos de vida de las mujeres migrantes.

Es relevante identificar que quienes principalmente han agredido a las mujeres que participan en este artículo son: parejas-exparejas, familiares, agentes del Instituto Nacional de Migración, agentes de la Guardia Nacional, y agentes de las policías municipales. Si bien la violencia se ha presentado a distintos niveles de intensidad dependiendo la situación y/o contexto, puntualizar en los responsables del miedo, ansiedad, estrés y tristeza que sufren las mujeres en la migración permite dirigir las propuestas de política pública e incidencia que emergen desde la sociedad civil de una mejor manera.

En ese sentido, para algunas usuarias la situación de la violencia ha sido aún peor en México que en el lugar de origen, en un contexto en el que la principal víctima han sido personas menores de edad, por lo que la preocupación y la carga emocional para la usuaria de IMUMI se multiplica, pues no solamente el temor es por lo que a ella le pudiera suceder.

$\mathrm{Su}$ hija fue violentada por un familiar por lo que presentó denuncia ante las autoridades correspondientes en el estado de Puebla en 2016. En ese mismo contexto, la usuaria acudió a terapia psicológica. La denuncia no procedió, no hicieron nada. El abuso psicológico continuó en México... Fue peor en México. (E55, Comunicación personal, febrero de 2020)

Cabe destacar que a partir del análisis cuantitativo que se realizó en una primera etapa del proyecto se detectó que de las mujeres atendidas por IMUM al menos el $72 \%$ fueron víctimas de violencia física ya sea en el lugar de origen o en el de destino. De esta forma, el trayecto de vida de las personas está inmerso en situaciones de alto grado de violencia, lo que indica que la ejecución del proyecto migratorio no precisamente elimina esta temática dentro del entorno en el que se desenvuelven las usuarias migrantes, pues la persecución y el miedo a ser alcanzada por quienes les agredieron en algún momento continúa siendo una constante. Incluso, aunado a la violencia de tipo físico, la violencia de tipo sexual se presentó de igual manera en algunos de los casos. 
La usuaria fue agredida por un hombre en Tapachula, quien abusó sexualmente de su hija. Por lo que se movió a otra ciudad. Ha tenido problemas con autoridades, particularmente con policías municipales en Tijuana, a donde se movió de nueva cuenta por miedo a persecución. Ha tenido que dejar el seguimiento a los trámites por motivos de seguridad; se ha movido por miedo; teme por su vida. (E41, Comunicación personal, noviembre de 2020)

A partir de lo que menciona E41 en su testimonio, se puede ilustrar como el desarrollo de los trámites vinculados a la solicitud de la condición de refugiada en México se ve interrumpida por las mismas consecuencias de la violencia e inseguridad que se presenta en la vida diaria de las personas, donde es reiterativo el papel de las autoridades policiacas adjudicándose la realización de tareas que rebasan su jurisdicción, es decir, los temas migratorios a pesar de que no corresponden a las corporaciones policiacas, estas últimas se han convertido en uno de los mayores obstáculos para la población migrante.

Esta experiencia se registró en el caso de E44, donde "la policía guatemalteca la intenta extorsionar y la golpea. Antes en la frontera en Guatemala y Honduras la secuestraron, pero escapó". De igual manera, en el contexto mexicano se han presentado este tipo de situaciones donde agentes policiacos han intervenido generando aún más complejidad a la situación de las mujeres migrantes, tal como lo indica el registro sobre la experiencia de E40, misma que “fue víctima de abuso sexual y física al momento de llegar a México. En Chiapas desconocidos abusaron de ella. En Veracruz unos policías la intimidaron pidiéndole sus documentos de identidad" (E40, comunicación personal, febrero de 2020).

El resultado de estas experiencias que fueron registradas a partir de los relatos de las mujeres migrantes muestra cómo el ejercicio de la violencia y las relaciones de poder desiguales generan una carga emocional que afecta sobremanera la estabilidad psicológica, lo cual, aunado a los problemas ya mencionados, colocan a las personas en movilidad en una posición de notable vulnerabilidad. En ese sentido, en el siguiente apartado se describe como la violencia de tipo psicológica ha estado presente en el trayecto de vida de las mujeres que aparecen en este artículo.

\section{"Fui víctima de asalto a mano armada. Me secuestraron"}

Si bien la violencia física es recurrente en los testimonios de mujeres migrantes atendidas por la Clínica Jurídica del IMUMI y hasta cierto grado más identificable por parte de las mismas víctimas, la violencia de tipo psicológica se presenta incluso en un grado mayor de frecuencia. En ese sentido, el $83.6 \%$ de las usuarias reportaron haber sufrido afectaciones psicológicas derivadas de las agresiones recibidas ya sea por parte de familiares y parejas o exparejas, o bien por autoridades migratorias y/o agentes policiacos.

Asimismo, las pandillas y el crimen organizado tanto en el lugar de origen como en el de destino han desempeñado un papel preponderante en la generación de situaciones de alto nivel de estrés, miedo y ansiedad dentro de los trayectos de vida de las usuarias. De esta forma lo relata E64, quien tuvo que salir de Honduras no solamente por la violencia física y psicológica, sino también patrimonial y económica que sus agresores ejercieron contra ella y su familia.

Prestamos dinero a un vecino que resultó estar ligado a pandillas y al crimen organizado, de lo que solo nos dimos cuenta cuando lo vimos usando el dinero que le préstamos para 
administrar una tienda de autos que vendía drogas a pandilleros. Cuando intentamos pedirle que nos devolviera el préstamo empezó a amenazarnos, lo que empeoró cuando fuimos a la corte a presentar una denuncia en su contra. Después de enterarse de que habíamos presentado una denuncia en su contra, incluso envió a hombres para intentar atropellarme en un automóvil. (E64, comunicación personal, octubre de 2020).

Un elemento importante que se resalta a partir de este testimonio se vincula a las consecuencias que derivan de las denuncias interpuestas en contra de los agresores, en un contexto en el que ni las autoridades pueden ayudar de manera integral y sistemática a las personas en movilidad, cuyo aspecto a destacar es que muchas de ellas carecen de redes de apoyo en el lugar de destino, lo cual representa un elemento crucial para el desarrollo de los proyectos migratorios (Portes \& Landolt, 2000).

Otro aspecto relevante en las relaciones de poder desiguales, cuyo efecto principal es que generan violencia de género, es el vinculado al reconocimiento de la misma violencia en sus distintos tipos y/o manifestaciones.

Dicho de otra manera, la falta de información en torno a lo que representa una violencia provoca que la persona que es víctima en ocasiones no reconozca que está siendo violentada por su agresor. Es así como se registró el caso de E60, quien a pesar de saberse violentada no tiene la certeza sobre qué tipos de violencia se han ejercido en su contra.

Aunque no reporta de forma explícita los tipos de violencia que ha sufrido, la usuaria se siente violentada. Ha sufrido de violencia verbal por parte de su esposo, esto luego de que la usuaria descubriera que él tenía otra relación. (E60, Comunicación personal, enero de 2020).

Si bien aquí se subraya la violencia de tipo psicológico, las relaciones que alrededor de esta misma se construyen es una parte importante para ejemplificar determinado tipo de violencia no puede ser entendida de manera aislada, sino que de forma relacional, pues a partir de esas mismas intersecciones se ilustra de buena forma cómo los entornos se vuelven más complejos generando escenarios cuya carga emocional se potencializa de manera significativa, como sucede en el caso de E58, quien salió de nicaragua huyendo de la violencia y dificultades económicas.

La usuaria sufrió las consecuencias de guerras civiles en el país de origen, donde jóvenes fueron asesinados, las oportunidades de empleo formal disminuyeron, y las amenazas de muerte eran una constante. Es por ello por lo que ella y otros miembros de su familia salieron de su país. (E58, Comunicación personal, octubre de 2020)

Aunado a todo lo anterior, la discriminación y el racismo aparecen recurrentemente en los testimonios de las personas que participaron en este artículo, incluso hay usuarias que reportaron haber sufrido de xenofobia por parte de sus parejas, quienes han recurrido a las amenazas, a la retención de documentos migratorios y/o pasaportes, lo que ha propiciado retrasos en los trámites ante las autoridades migratorias mexicanas.

Es su propia expareja quien principalmente la violentaba de distintas formas. Recibió humillaciones y maltratos por parte de él mismo. Su expareja la humillaba y discriminaba mencionándole que era una inmigrante que no valía nada. Lo que generaba en la usuaria problemas psicológicos. (E56, Comunicación personal, enero de 2021)

$\mathrm{Si}$ bien las redes migrantes sirven como un apoyo para quienes dejan su lugar de origen para buscar mejorar su calidad de vida en otro territorio, 
en el caso de los agresores sirven precisamente para generar obstáculos que impidan el desarrollo del proyecto migratorio. En ese sentido, E55 enfrentó situaciones complejas debido a las redes con las que contaba su esposo.

Sufrió de violencia psicológica por parte de su esposo. Recurrentemente él la amenaza con quitarle a las niñas, lo que le genera a la usuaria gran temor debido a las redes con las que cuenta su esposo por ser militar. Aunque no hubo denuncia por la violencia ejercida, la misma usuaria reportó ante el jefe militar de su esposo. (E55, Comunicación personal, febrero de 2020)

Esta forma de ejercer la violencia psicológica se repite de forma significativa entre las historias registradas por el equipo de la Clínica Jurídica, donde es común que la persona que ejerce la violencia sea parte de la misma estructura familiar, ya sea esposo, pareja o incluso la misma familia extendida de la víctima. En este contexto, fue también común que las mujeres mencionaran como prioridad el bienestar de sus hijos/hijas, aunque en algunos de los casos los menores se habían quedado en el lugar de origen con la abuela o algún pariente cercano. No obstante, en algunos casos las usuarias estaban embarazadas y preocupadas ante la incertidumbre en la que se encontraban.

El padre, cuando está enojado, la amenaza de que le va a quitar al bebé, pues ella no tiene derechos ni papeles en México. Ella tiene temor a que esto pase, a que cuando tenga el bebé él llegue al hospital y se lo lleve. Quiere saber si puede registrar al bebé solo con el nombre de ella. Cómo puede asegurar proteger a su bebé, pues el padre es violento. nunca llamó a la policía en esos episodios. (E58, comunicación personal, octubre de 2020)

A partir de los casos presentados en este artículo es posible señalar que la violencia en contra de las mujeres en movilidad se ha agravado durante los últimos años, lo que obliga a las autoridades a diseñar y ejecutar políticas públicas que no solamente les permitan realizar sus trámites y permanecer en el país de forma legal, sino también asegurar que su integridad tanto física como psicológica no será quebrantada por ninguno de los actores involucrados que se mencionan en este artículo. Es así como después del análisis presentado en esta sección, se describen algunas de las principales conclusiones.

\section{CONCLUSIONES}

De esta forma, pensar que la violencia se presenta de manera aislada con relación a los distintos tipos existentes sería incurrir en una reflexión simplista y reduccionista en torno a la problemática que enfrentan las mujeres en México. Las mujeres migrantes son víctimas no solamente de un tipo de violencia, sino que pueden presentarse distintas prácticas en su trayecto de vida que las enfrenta a otras violencias que, como se mencionó ya, pueden pasar desapercibidas debido al desconocimiento, por ello también la importancia de los gobiernos, sociedad civil y academia de empoderar a la mujer brindándoles información que les permita identificar los momentos en los cuales están siendo víctimas de algún abuso, como podría ser, por ejemplo, la retención del pasaporte (violencia patrimonial) en el aeropuerto por agentes de migración.

Es así como los hacedores de políticas públicas, organizaciones de la sociedad civil y academia deben considerar estas relaciones en sus propuestas para mejorar la calidad de vida de esta población, donde la perspectiva de género y la interseccionalidad son fundamentales debido a que permiten incluir categorías temáticas que actualmente se quedan fuera de los lineamientos y programas que 
tienen como propósito proteger a las personas que huyen de sus países por motivos de violencia y/o persecución.

Hacerlo de forma contraria generaría una reproducción constante en la segmentación de la problemática, cuya implicancia sería la solución del problema de manera parcial y no integral como lo demandan los tratados internacionales a los que se adhiere México en materia de migración y derechos humanos.

Una de las propuestas que emerge del análisis de coocurrencia de violencia se relaciona a la necesidad de reforzar el acompañamiento tanto psicológico y jurídico que se les brinda a las mujeres solicitantes de asilo debido a que la violencia no solamente se relaciona entre sus distintos tipos, sino que las acompaña desde el lugar de origen al de destino, transnacionalizando las prácticas violentas que les obligaron a dejar su hogar.

En definitiva, se trata de una tarea importante que quienes se encuentran involucrados en el tema de la migración internacional en México deben abordar de manera urgente ante el aumento de los flujos de personas, lo cual se modifica de forma constante a partir de las políticas migratorias que implementan principalmente los Estados Unidos, por lo que es también crucial adoptar una postura metodológica y teórica en materia de migración que sea igual de flexible que el fenómeno mismo, de lo contrario la ola migratoria rápidamente rebasará a los actores involucrados en el tema, ya sean funcionarios de los distintos tipos de gobierno, instituciones de investigación y organizaciones de la sociedad civil.

Algunas de las usuarias han mostrado inseguridad por la persecución que siguen viviendo en México, pero también una inseguridad por ser mujeres, por no encontrar recursos que puedan ayudarles a ellas y sus familias a poder insertarse en la sociedad mexicana, lo que también vulnera su estabilidad e inserción en el país, a fin de que puedan fortalecer sus herramientas personales, reconocer la violencia y desligarse de ella para un bien propio y de sus familias.

Es importante mencionar que la violencia se reproduce, transforma y alimenta de las mismas prácticas que se ejecutan dentro de la estructura social, es por ello que urgen cambios significativos en los distintos niveles, lo que sin duda beneficiaría no solamente a las personas en movilidad, sino a toda la sociedad civil que actualmente se rehúsa a "abrir las puertas" a personas originarias de distintas latitudes.

\section{BIBLIOGRAFÍA}

Comisión Mexicana de Ayuda a Refugiados. (2021). La COMAR en Números. https://www.gob. $\mathrm{mx} / \mathrm{comar} /$ articulos/la-comar-en-numeros-273823?idiom=es

Kuckartz, U. (2014). Qualitative Text Analysis A Guide to Methods, Practice and Using Software (1st ed.). Sage Publications.

Landeros Jaime, F. (2020). Conversión y transmisión de capital en un contexto migratorio: etnografía con familias migrantes en Chile. Migraciones Internacionales, 11(22), 1-22. https:// doi.org/2594-0279 https://doi.org/10.33679/ rmi.v1i1.2094

Portes, A., \& Landolt, P. (2000). Social Capital: Promise and Pitfalls of its Role in Development. Journal of Latin American Studies, 32(2), 529-547. https://doi.org/10.1017/ S0022216X00005836

Rädiker, S., \& Kuckartz, U. (2019). Analyzing MAX- 
QDA Data with Qualitative Text, Audio, and Video. In Springer (1st ed.). Springer.

Torre Cantalapiedra, E. (2020). Destino y asentamiento en México de los migrantes y refugiados centroamericanos. Revista Trace, 77, 122-145. http://trace.org.mx/index.php/trace/article/view/726

Torre Cantalapiedra, E., \& Mariscal Nava, D. M. (2020). Batallando con fronteras: estrategias migratorias en tránsito de participantes en caravanas de migrantes. Estudios Fronterizos, 21, 1-21. https://doi.org/10.21670/ ref. 2005047

Torre Cantalapiedra, E., París Pombo, M. D., \& Gutiérrez López, E. E. (2021). El sistema de refugio mexicano: entre proteger y contener. Frontera Norte, 33, 1-26. 